\title{
Proteomic analysis of psoriatic skin tissue for identification of differentially expressed proteins: Up-regulation of GSTP1, SFN and PRDX2 in psoriatic skin
}

\author{
JOOHYUN RYU ${ }^{1}$, SUNG GOO PARK ${ }^{1}$, BYOUNG CHUL PARK ${ }^{1}$, \\ MISUN CHOE ${ }^{2}$, KYU-SUK LEE ${ }^{3}$ and JAE-WE CHO ${ }^{3}$ \\ ${ }^{1}$ Medical Proteomics Research Center, KRIBB, Daejeon 305-333; Departments of ${ }^{2}$ Pathology and ${ }^{3}$ Dermatology, \\ Keimyung University School of Medicine, Daegu 700-712, Republic of Korea
}

Received May 23, 2011; Accepted June 24, 2011

DOI: $10.3892 / \mathrm{ijmm} .2011 .757$

\begin{abstract}
Psoriasis is a chronic inflammatory skin disease, characterized by a combination of abnormal proliferation of keratinocytes, immunology and vascular proliferation. Proteomic analyses have revealed some clues regarding the pathogenesis of psoriasis. In the present study, we conducted an investigation of different proteomes of psoriatic lesional skin, and compared them with those of normal and nonlesional psoriatic skin. We performed 2-D gel electrophoresis, liquid chromatography tandem mass spectrometry (LC-MS/ MS) analysis and database searches. Expression of proteins were evaluated by immunoblot and immunohistochemistry analyses. Our data showed differential expression of 74 and 145 protein spots in non-lesional and lesional psoriatic skin, respectively. Eleven of 36 proteins, which were identified by LC-MS/MS, were categorized as apoptosis-regulating proteins. Other protein spots were categorized as proteins with involvement in the negative regulation of apoptosis, defense response-related proteins and inflammatory response. Of particular interest, increased expression of glutathione $\mathrm{S}$ transferase 1 (GSTP1) and peroxiredoxin 2 (PRDX2), which are involved in the Redox balance system, and SFN, which is involved in the cellular proliferation system, was observed in psoriatic lesional skin. Localization of GSTP1 and SFN was observed above the middle layer of the epidermis in psoriatic skin lesions. Expression of PRDX2 was clearly observed below the middle layer of the epidermis in chronic type psoriatic skin lesions. Taken together, 36 identified proteins were associated with biological regulation, including regulation of cell death, defense response, inflammatory response and reactive oxygen species (ROS) regulation. PRDX2 and GSTP1 may play roles
\end{abstract}

Correspondence to: Dr Jae-We Cho, Department of Dermatology, Keimyung University School of Medicine, 194 DongSan-Dong Jung-Gu, Daegu 700-712, Republic of Korea

E-mail: janylove99@dsmc.or.kr

E-mail: janylove99@hanmail.net

Key words: psoriasis, proteomics, proteomes in compensating mechanisms for reduction of ROS stress, and SFN may play roles in prevention of cancer development in proliferating cells through $\mathrm{G} 2 / \mathrm{M}$ cell cycle arrest upon accidental DNA damage within psoriatic skin lesions.

\section{Introduction}

Psoriasis vulgaris is one of the most prevalent chronic inflammatory skin diseases $(1,2)$. Psoriasis is a complex disease affecting cellular, gene, and protein levels, and is presented as scaly plaques on the body $(3,4)$. Skin lesions are characterized by abnormal keratinocyte differentiation, hyperproliferation of keratinocytes, and infiltration of inflammatory cells (5-7). Psoriatic keratinocytes were found to be intrinsically growth dysregulated by several mechanisms. Keratinocytes of psoriatic skin reached the surface of the skin from the basal layer in as few as 6-8 days, compared with approximately 40 days in normal skin. In addition, keratinocytes in psoriasis lesions produced an array of proteins that attract leukocytes, including S100 proteins (A7, A8, A9, A12) $(8,9)$. Once inflammation is initiated by keratinocyte products, leukocyte recruitment or inflammation is amplified by cytokine networks, and several reactive oxygen species (ROS) are produced by keratinocytes and inflammatory cells $(10,11)$. Compelling evidence demonstrating that ROS-mediated oxidative stress could contribute to the pathogenesis of psoriasis has been reported $(11,12)$.

In this study, we compared the differential protein expression between normal $(\mathrm{N})$, non-lesional psoriatic skin lesions (NP), and lesional psoriatic skin lesions (LP) using twodimensional gel electrophoresis and liquid chromatography tandem mass spectrometry (LC-MS/MS) analysis.

\section{Materials and methods}

Materials. Antibodies against peroxiredoxin 2 (PRDX2), SFN, and glutathione S transferase 1 (GSTP1) were obtained from the Korea Research Institute of Bioscience and Biotechnology (Abnova, Taiwan).

Patients. Five healthy volunteers and 40 psoriatic cases were enrolled for the study. The inclusion criteria were the mani- 
festation of well demarcated, erythematous, scaly psoriatic plaques on the trunk and extremities. Study subjects did not use any systemic anti-psoriatic treatments for 2 weeks before skin biopsy. Informed consent was obtained from all subjects, under protocols approved by the Investigational Review Board of the Dongsan Hospital of Keimyung University. Two biopsies were taken from each patient; one was obtained from lesional skin of patients and the other from uninvolved skin. Immediately upon removal, biopsies were snap-frozen in liquid nitrogen and stored at $-80^{\circ} \mathrm{C}$.

Two-dimensional gel electrophoresis. Two-dimensional gel electrophoresis (2-DE) was performed using isoelectric focusing (IEF) and SDS-PAGE analysis. Dry-strips were rehydrated with 150 or $500 \mu \mathrm{g}$ of protein in solubilization solution $(9 \mathrm{M}$ urea; 2\% CHAPS; $4 \mathrm{M}$ thiourea; $2 \%$ IPG buffer, $\mathrm{pH}$ 4-7; 18 mM DTT and a trace of bromophenol blue) and loaded onto Immobiline Drystrips. Rehydration proceeded for $12 \mathrm{~h}$ at room temperature and IEF was conducted at $20^{\circ} \mathrm{C}$ in a gradient mode for $1 \mathrm{~min}$ at $300 \mathrm{~V}, 90 \mathrm{~min}$ at $3500 \mathrm{~V}$ and $10 \mathrm{~h}$ at $3500 \mathrm{~V}$. Following IEF separation, the dry-strips were equilibrated for $30 \mathrm{~min}$ in an equilibration buffer (50 mM Tris- $\mathrm{HCl} \mathrm{pH} 6.8,6 \mathrm{M}$ urea, 30\% glycerol, 2\% SDS and a trace of bromophenol blue). We then added $0.25 \%$ DTT to the first equilibration buffer, and replaced DTT with $4.5 \%$ iodoacetamide in the second equilibration buffer. The second dimension of the separation was carried out on a SDS polyacrylamide vertical slab gel. After 2-DE, proteins were visualized by silver staining according to the manufacturer's protocol (GE Healthcare).

Image analysis. The 2-DE images were analyzed using the Progenesis SameSpots program, v2.0. (Nonlinear Dynamics, Nonlinear USA).

Destaining and in-gel trypsin protein digestion and extraction. Protein spots separated by $2-\mathrm{DE}$ were excised manually from 2-DE gels and destained using oxidation buffer $(15 \mathrm{mM}$ potassium ferricyanide and $50 \mathrm{mM}$ sodium thiosulphate). Destained gels were re-swelled and dehydrated using $100 \mu \mathrm{l}$ of $100 \mathrm{mM}$ ammonium bicarbonate in 50\% acetonitrile. The gels were re-swollen and dehydrated for an additional two to three times, then dried. The dried gels were digested by $50 \mathrm{mM}$ ammonium bicarbonate, $\mathrm{pH} 8$, containing $0.05 \mu \mathrm{g} / \mu \mathrm{l}$ trypsin for $16 \mathrm{~h}$ at $37^{\circ} \mathrm{C}$. The tryptic peptides were extracted three times to recover all of the peptides from the gel particles. The recovered peptides were concentrated by drying the final volume of the extracts in a vacuum centrifuge. The concentrated peptides were then mixed with $20 \mu \mathrm{l}$ of $0.1 \%$ formic acid in $3 \%$ acetonitrile in preparation for LC-MS/MS analysis.

LC-MS/MS analysis and database searches. Nano LC of tryptic peptides was performed using a Waters Nano LC system equipped with a Waters C18 Nano Column. Samples were loaded onto the column. Peptides were eluted from the column with a gradient ranging from 2 to $40 \%$ binary solvent $\mathrm{B} 1$ for $45 \mathrm{~min}$ at $0.3 \mu \mathrm{l} / \mathrm{min}$. Mass spectrometry analysis of tryptic peptides was performed using a Waters Synapt ${ }^{\mathrm{TM}}$ HDMS. The mass spectrometer was operated in V-mode for all measurements. All analyses were performed using a positive mode Nano ESI with a NanoSpray source. Accurate mass
LCMS data were collected via a Data Dependent Acquisition (DDA) mode of acquisition. Continuum LC-MS/MS data were processed and used in database searches using the Protein Lynx Global Server (PLGS), version 2.3 (Waters). Ion detection, clustering and normalization were performed using PLGS. Processed data were used to search the UniProtKB (Homo sapiens). Processed ions were sequenced and mapped against the UniProtKB (Homo sapiens) using the PLGS, v2.3 and Mascot Daemon programs (http://www.matrixscience. com).

Immunoblot analysis. Tissues were prepared in lysis buffer [10 mM Tris (pH 7.4), 5 mM EDTA, $130 \mathrm{mM} \mathrm{NaCl}, 1 \%$ Triton $\mathrm{X}-100$, phenylmethylsulphonyl fluoride (PMSF, $10 \mathrm{mg} / \mathrm{ml}$ ), aprotinin $(10 \mathrm{mg} / \mathrm{ml})$, leupeptin $(10 \mathrm{mg} / \mathrm{ml}), 5 \mathrm{mM}$ phenanthroline and $28 \mathrm{mM}$ benzamidine- $\mathrm{HCl}]$. The protein concentration of extracts was estimated with Bradford reagent (Bio-Rad, Hercules, CA, USA) using bovine serum albumin as the standard. Equal amounts of protein ( $40 \mu \mathrm{g} / \mathrm{lane})$ were resolved by 6.5-12\% sodium dodecyl sulfate-polyacrylamide gel electrophoresis, and transferred onto a nitrocellulose membrane. The membrane was incubated with respective specific antibodies such as PRDX2, SFN, and GSTP1 protein. The membrane was continuously incubated with appropriate secondary antibodies coupled to horseradish peroxidase, and developed by the ECL Western detection reagents (Amersham Pharmacia Biotech, Piscataway, NJ).

Immunohistochemistry. Formalin-fixed and paraffin-embedded tissue specimens were cut on a microtome into $5 \mu \mathrm{m}$ sections. The sections were deparaffinized in xylene and hydrated in alcohols of decreasing concentration. In order to visualize the antigen, the sections were heated in citrate buffer $\mathrm{pH}$ 6.0. After cooling to room temperature, in order to block endogenous peroxidase activity, 5 min incubation was performed with $\mathrm{H}_{2} \mathrm{O}_{2}$. After rinsing in PBS, pH 7.4, for $15 \mathrm{~min}$, and blocking with the antibody diluent (Golden Bridge, Mukilteo, Washington, USA) for $5 \mathrm{~min}$, the sections were incubated with antibodies: PRDX (monoclonal antibody, Abnova) in a dilution 1:500 for $2 \mathrm{~h}$; SPN (polyclonal antibody, Abnova) in a dilution of 1:400 for $2 \mathrm{~h}$ and GSTP1 (polyclonal antibody, Abnova) of 1:400 for $2 \mathrm{~h}$ at $37^{\circ} \mathrm{C}$. In the next step, biotinylated antibody was used in the reaction and then the streptavidin-peroxidase complex $\left(\mathrm{LSAB}^{\circledR}\right.$ + System-HRP, Dako) was applied. The antigen-antibody complex was visualized by DAB chromogen.

\section{Results}

Proteomic analysis of psoriatic skin tissue for identification of differentially expressed proteins and functional classification of identified proteins. Proteomic analysis was performed for investigation of differentially expressed proteins between $\mathrm{N}$, NP and LP skin. Total protein extracts obtained from each skin sample were separated by 2 -DE and visualized by silver nitrate staining. Differences in protein profiles were compared using the Progenesis SameSpots program v2.0. In conduct of our research, two methods were used for identification of psoriasis-associated proteins. First, proteome differences in LP skin, compared with N and NP skin, were individually analyzed in 8 patients. We observed the different proteomes 
A

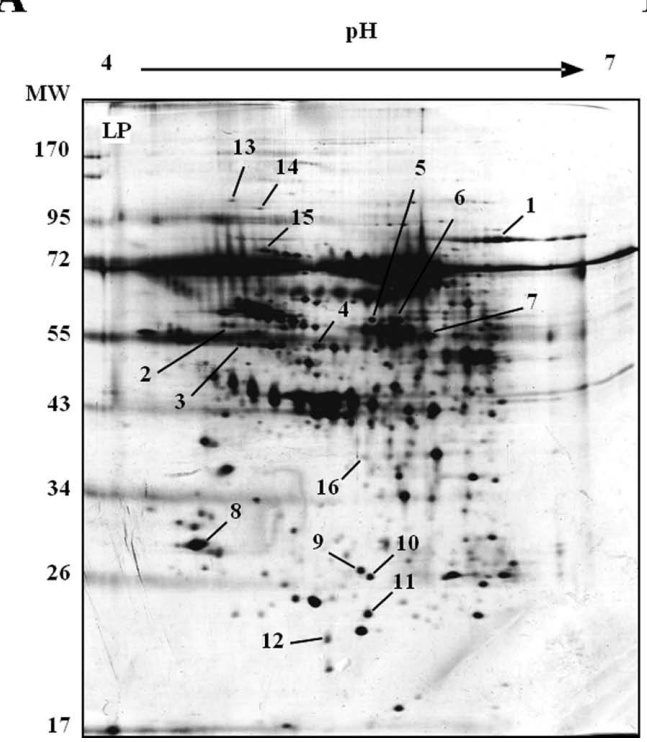

B

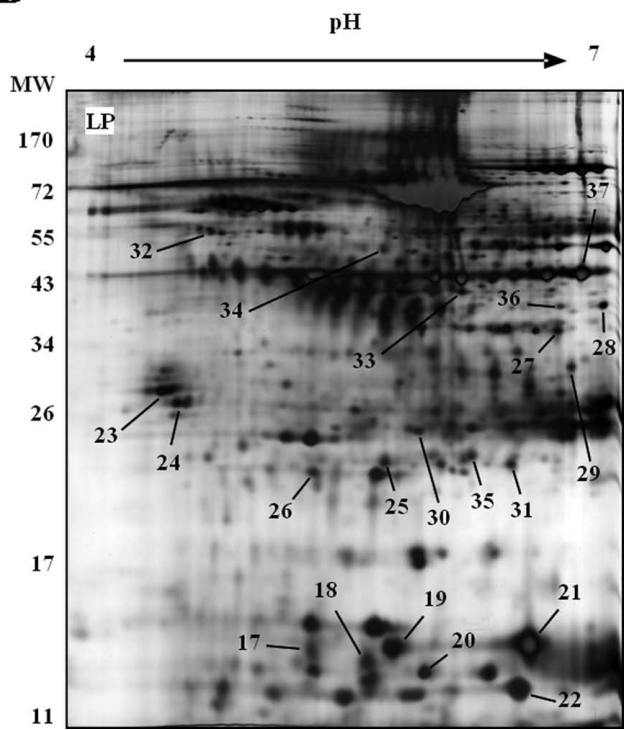

Figure 1. 2-DE gel images of lesional psoriatic (LP) skin. (A and B) Representative gels showing proteome differences in LP, compared with normal (N) and non-lesional psoriatic (NP) skin lesions, in 8 patients and in total protein from 28 pooled patients, respectively. Thirty-seven spots, marked in (A and B), indicate the up-regulated proteins in LP skin. These spots were identified by LC-MS/MS, and are listed in Table I.

A

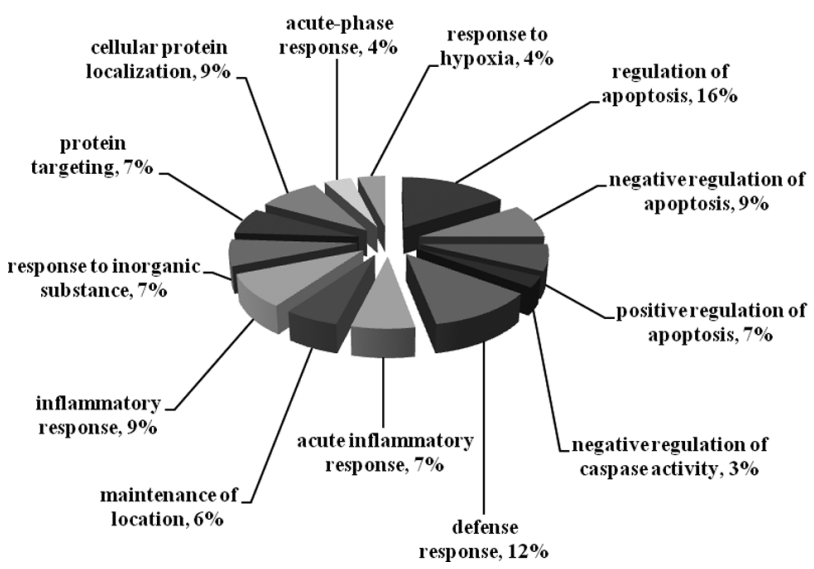

B

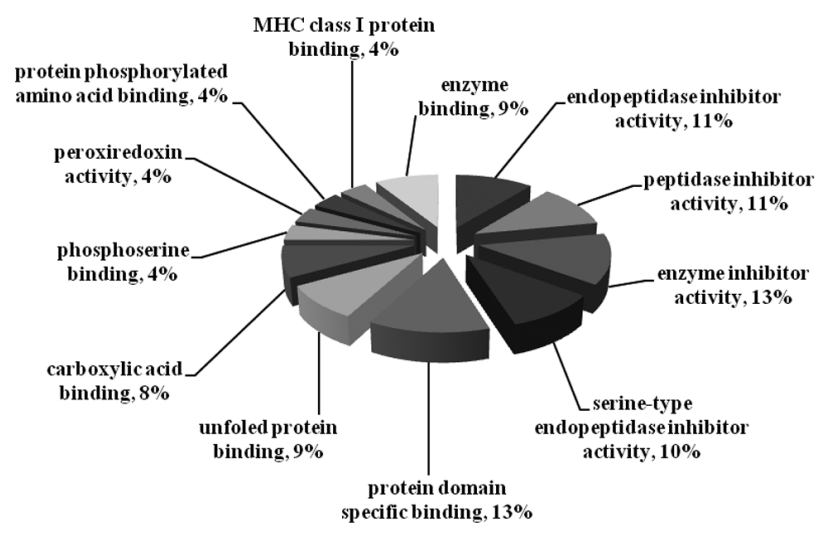

Figure 2. Classification of up-regulated proteins in lesional psoriatic skin by proteomic analyses. A total of 36 up-regulated proteins were categorized according to biological process (A) and molecular function (B) using information from the Gene Ontology database (http://www.geneontology.org). Percentages denote the proportion of the 36 proteins in each group.

among patients (data not shown). A representative gel of LP skin shows the differentially expressed protein spots, compared with NP skin (method A, Fig. 1A). Second, total proteins of 28 patients were pooled and analyzed for enrichment of lower level proteins and reduction of variation by clinical condition (method B, Fig. 1B). As expected, more protein spots were visualized through pooled proteome analysis. Approximately 600 and 1,000 protein spots were mapped in 2-DE gels. Individual and pooled proteome analysis showed differential expression of 74 and 145 protein spots in NP and LP skin, respectively. Of particular interest, most of the proteins were up-regulated in LP skin.

For identification of up-regulated protein, spots that showed more than a 2-fold increase were included for further analysis. In addition, we considered the clinical condition of each patient, and also included protein spots that showed irregularity, but a relatively increasing pattern. A total of 74 up-regulated spots were excised from 2-DE gels obtained using methods A and B, and analyzed by LC-MS/MS. Among them, 37 up-regulated spots were identified in psoriatic skin, and are listed in Table I. In particular, protein S100-A7 (S100A7), known as psoriasin, was reported as a highly up-regulated protein in psoriatic skin (8). This result is consistent with our observations from the pooled proteome analysis, and also indicates that our analytic methods were well established.

Thirty-six up-regulated proteins were assigned to functional categories according to biological process and molecular process using information from the Gene Ontology database (http://www.geneontology.org). As expected, in the analysis by biological process, 25 of 36 identified proteins were found to be associated with biological regulation, including regulation of cell death (apoptosis), defense response, inflammatory 


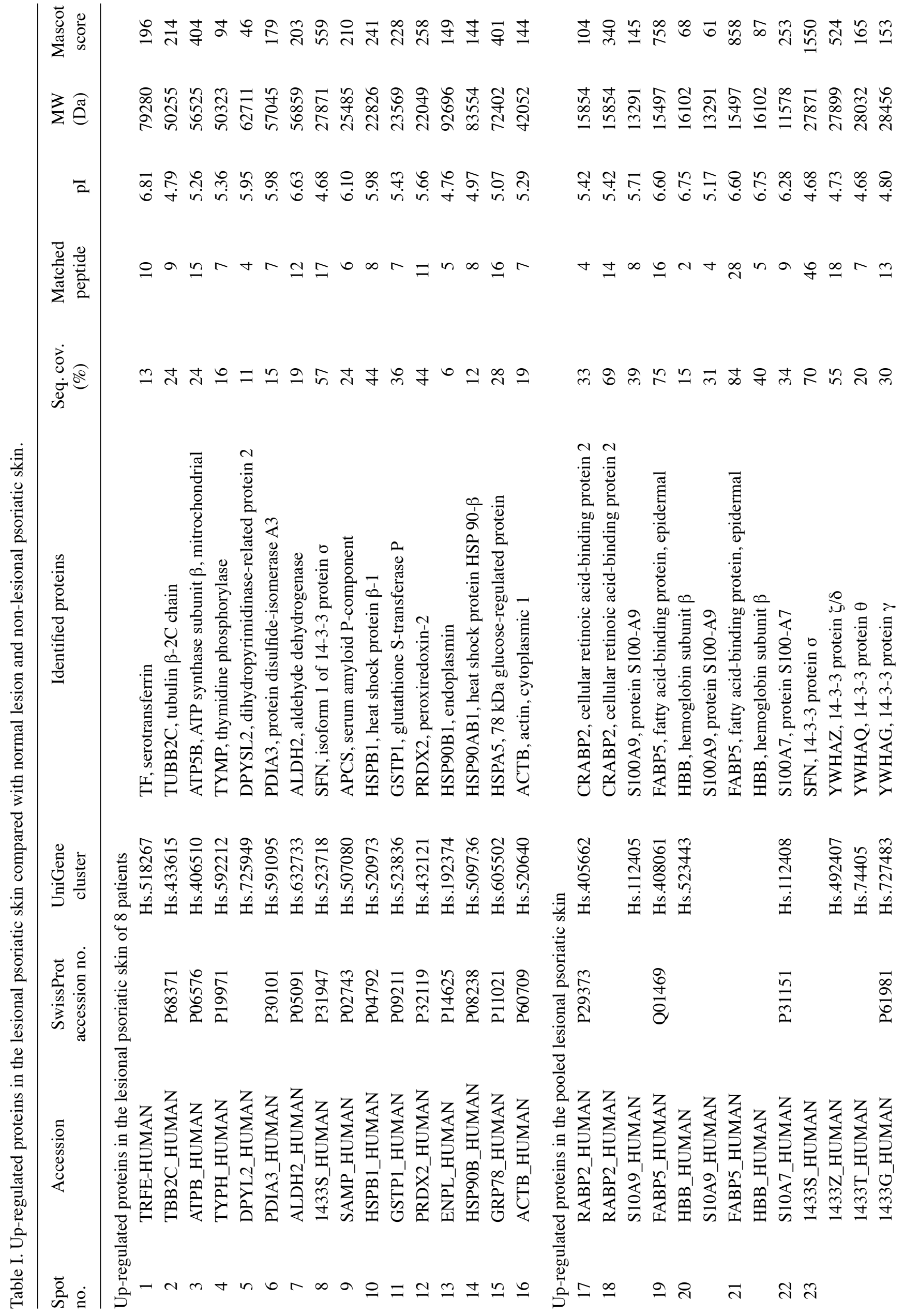




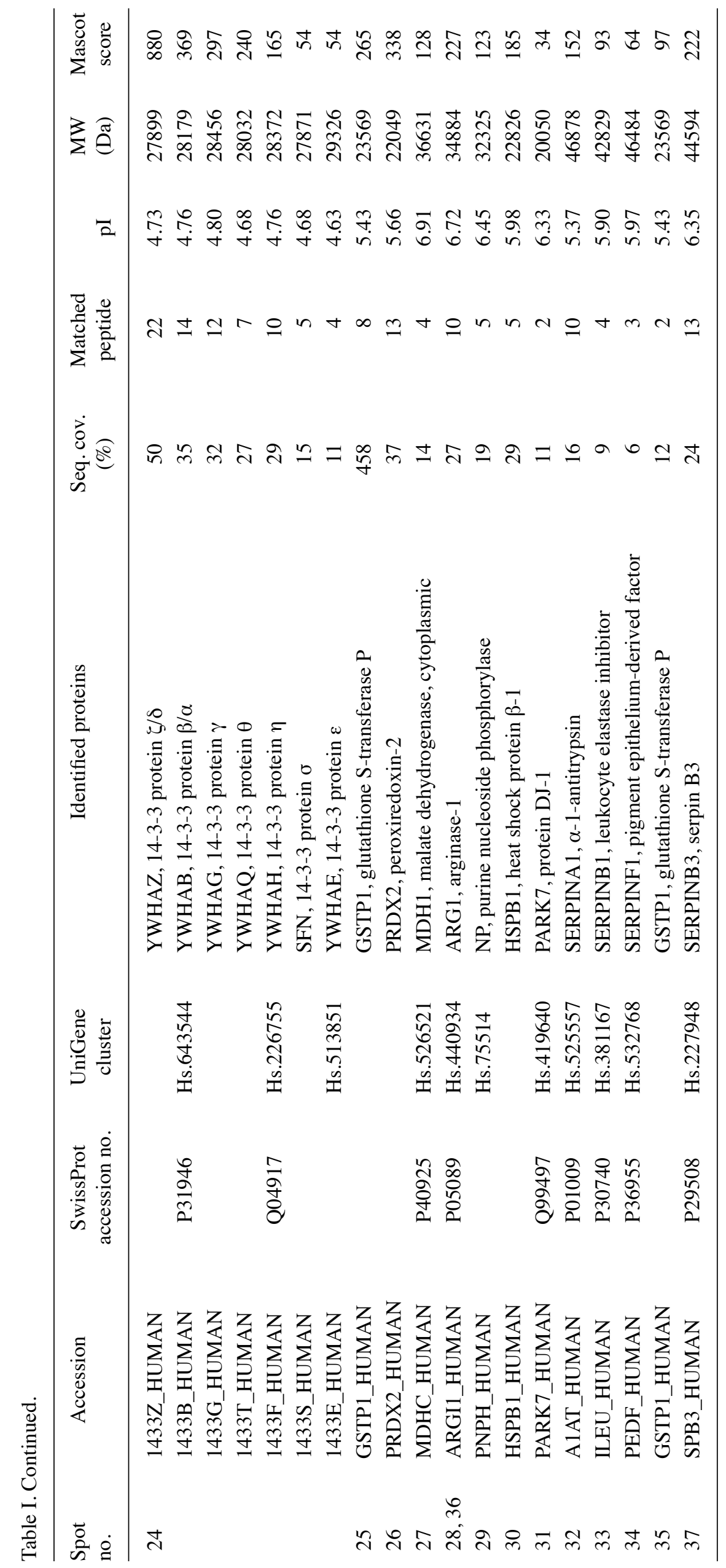


A

\section{GSTP1}
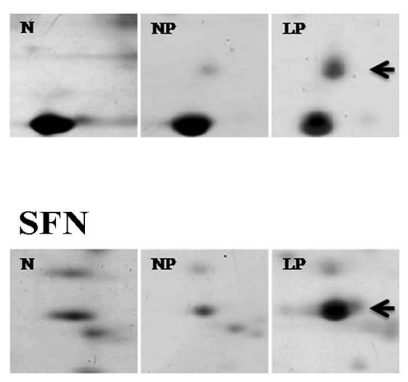

RPDX2

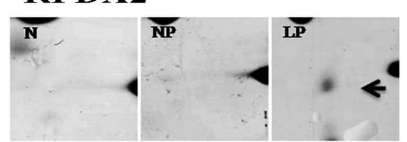

B

\section{GSTP1}

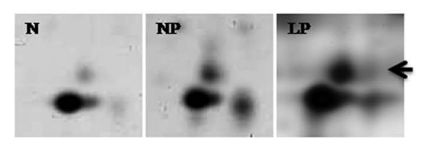

SFN

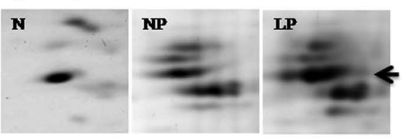

RPDX2

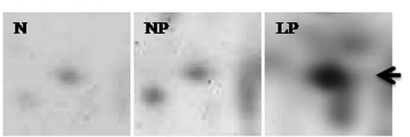

Figure 3. Representative features of GSTP1, SFN and PRDX2 between normal lesion (N), non-lesional psoriatic (NP) and lesional psoriatic (LP) skin. (A and B) Representative gels showing increased protein levels of GSTP1, SFN and PRDX2 in LP, compared with N and NP, in 8 patients and in pooled protein, respectively.

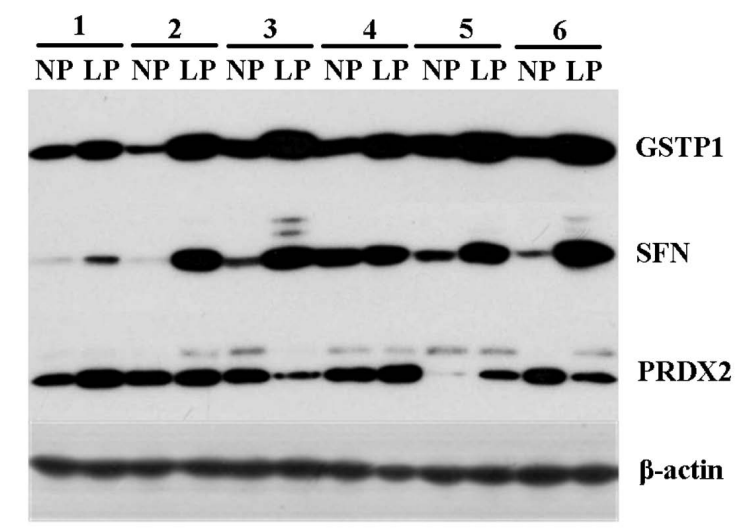

Figure 4. Expression of GSTP1, SFN and PRDX2 in psoriatic skin lesions. Proteins were extracted from non-lesional (NP) and lesional psoriatic skin (LP), and expression of GSTP1, SFN and PRDX2 was determined by Western blot analysis. Similar results were shown in two different experiments.

response, and so on (Fig. 2A). Eleven of 36 proteins were categorized as apoptosis-regulating proteins. Among them, 6 and 5 proteins were related to the negative regulation of apoptosis (GSTP1, HSPB1, HSP90B1, HSPA5, PRDX2 and YWHAZ) and to the positive regulation of apoptosis (PDIA3, YWHAE, SFN, TUBB2C and YWHAB), respectively. Eight of 36 proteins were defense response-related proteins (S100A7, S100A9, APCS, PRDX2, SERPINA1, TF, TUBB2C and YWHAZ) and some of the 8 proteins were also included in the inflammatory response category (S100A9, APCS, PRDX2, SERPINA1, TF and YWHAZ). In addition, more proteins with enzyme activity and protein binding activity were highly up-regulated in psoriatic skin (Fig. 2B).

Increased levels of ROS and hypoxia-induced factor have also been suggested in the pathogenesis of psoriasis $(13,14)$. In addition, findings from recent studies have indicated that an insufficiency of antioxidants or imbalance between oxidants

A

\section{GSTP1}
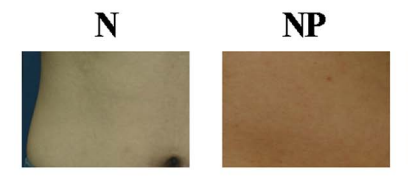

LP-1

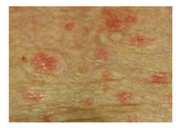

LP-1
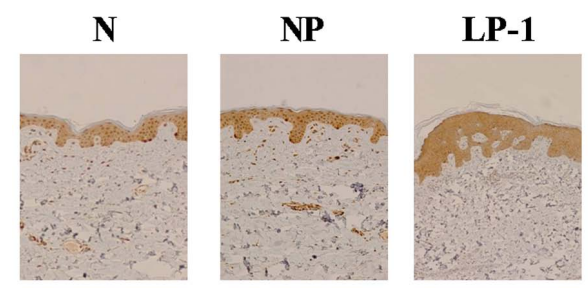

LP-2

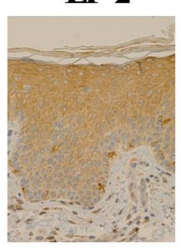

B
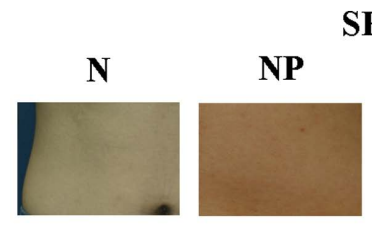

SFN

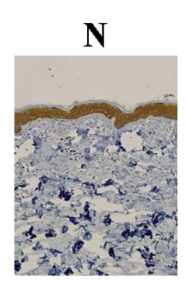

NP

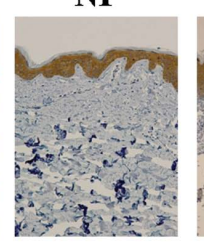

LP-1

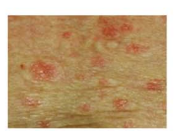

LP-2

C
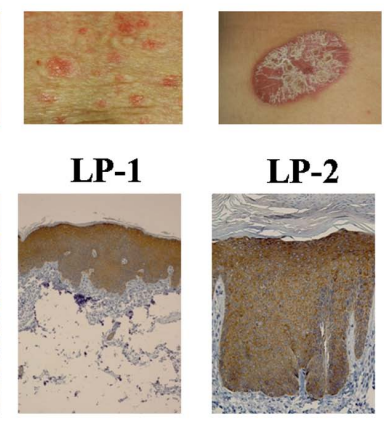

LP-2

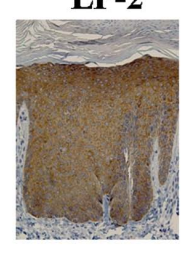

PRDX2
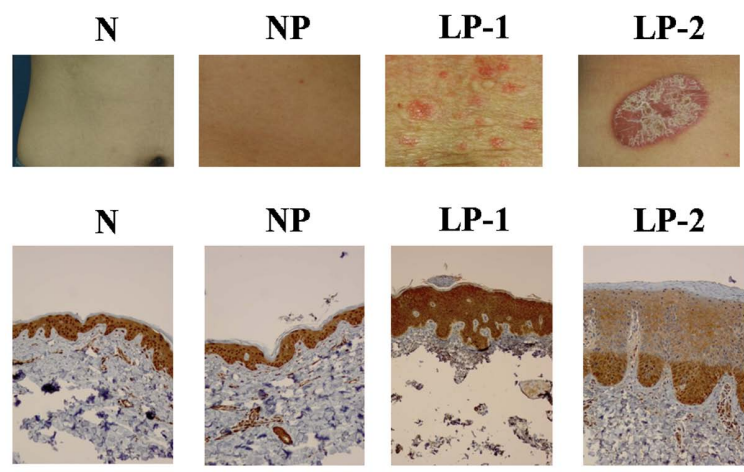

LP-1
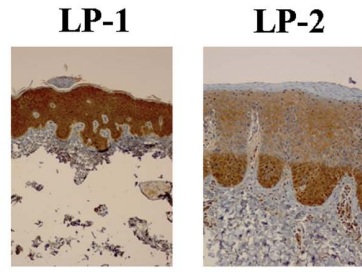

Figure 5. Immunohistochemical localization of GSTP1, SFN and PRDX2 in normal (N), non-lesional skin (NP), and lesional psoriatic skin (LP). (A and B) Expression of GSTP1 and SFN was usually detected in the middle and upper layers of the epidermis in LP; however, similar expression patterns were observed in N and NP. (C) Expression of PRDX2 was clearly observed in the lower layers of the epidermis in LP-2 (chronic plaques). Original magnification, $\mathrm{x} 200$.

and antioxidants may have a role in the pathogenesis of psoriasis $(12,15,16)$. However, the process is not completely understood. Therefore, based on our results and hypothesis, we were interested in the cellular metabolism of psoriatic skin, and focused on the 3 proteins PRDX2, GSPT1 and SFN (Fig. 3). These identified spots were further confirmed by performance of immunoblotting using GSTP1, SFN and PRDX2 antibodies. Increased expression of GSTP1 and SFN was clearly observed in LP; however, expression of PRDX2 
was not constantly up-regulated or down-regulated in LP, compared with NP (Fig. 4).

Expression patterns of GSTP1, SFN and PRDX2 in psoriasis. We studied the specific localization of GSTP1, SFN and PRDX2 in psoriatic lesional tissues by immunohistochemistry. Clinical pictures of acute and large chronic plaques of psoriasis were described as LP-1 and LP-2, respectively. Overexpression of GSTP1 and SFN was observed above the middle layer of the epidermis in LP-1 and LP-2 (Fig. 5A and B). Of particular interest, compared with $\mathrm{N}$ and $\mathrm{NP}$, overexpression of PRDX2 was clearly observed below the middle layer of the epidermis in LP-2; however, in the case of LP-1, increased expression of PRDX2 was observed in the entire epidermis (Fig. 5C).

\section{Discussion}

In general, pathogenesis of psoriasis presents as epithelial hyperplasia, Th1 cell-mediated inflammation, and angiogenesis (17-20). Consequently, we hypothesized that expression of up-regulated proteins in the epithelium may occur in association with cellular defense or survival mechanisms.

In the present study, we report that the proteome of LP indicates the up-regulation of proteins involving regulation of cell death (apoptosis), defense response, and inflammatory response (Table I). Expression of PRDX2, GSTP1 and SFN showed an increase in LP, compared with N and NP. Of particular interest, localization of PRDX2, GSTP1 and SFN differed according to the clinical lesional state of psoriasis. However, precise molecular mechanisms and the biological meaning concerning the different localization of these proteins in psoriasis will require further study.

In human cells, seven different SFN proteins regulate diverse cellular processes through binding to proteins with numerous functions. Expression of SFN is directly induced by p53 after DNA damage (21). Elevated levels of SFNs enforce $\mathrm{G} 2 / \mathrm{M}$ cell cycle arrest by sequestration of $\mathrm{Cdc} 2 /$ cyclin $\mathrm{B} 1$ complexes in the cytoplasm and are required for a stable $\mathrm{G} 2 / \mathrm{M}$ arrest after DNA damage $(21,22)$. Even though epidermal hyperplasia was observed in psoriasis, squamous cell carcinoma (SCC) was not found to occur naturally in psoriatic skin lesions, and SFN may play a role in prevention of development of SCC from psoriasis. In addition, SFN could prevent apoptosis by cytoplasmic sequestration of the pro-apoptotic protein, Bax (23). Thus, up-regulation of SFN in psoriasis may play a role in epidermal hyperplasia through prevention of apoptosis and in the prevention of carcinogenesis through maintenance of genome stability by cooperation with p53.

ROS, acting as second messengers, are known to influence cellular signal transduction pathways, such as proinflammatory signaling pathways. The most significant effects are observed in the MAPK/AP-1, NF- $\mathrm{B}$ and JAK-STAT signaling pathways, which have been regarded as early events in the inflammatory process in psoriasis $(11,13,15)$. Many systemic and lesionrestricted signs of severe oxidative stress can be observed in patients with active psoriasis (12). In the plasma and red blood cells of patients with active psoriasis, increased levels of malonyl dialdehyde (MDA) were interpreted as the fingerprint of the exhaustion of natural enzymatic and non-enzymatic antioxidant defenses, and, consequently, the prevalence of deleterious peroxidative processes in the cell membranes and plasma lipids of circulating cells $(24,25)$. Taken together, these results support the notion that an imbalance in the oxidantantioxidant system can be observed in psoriatic patients (26). In skin lesions, massive infiltration of various leukocyte populations in an activated state certainly leads to local release of a number of pro-oxidative species, which, in turn, are implicated in proinflammatory activation of the resident cells of the skin, particularly keratinocytes and fibroblasts. PRDX2 and GSTP1 play key roles in Redox-balance in proliferating cells. In this study, our data clearly showed that PRDX2 and GSTP1 were increased in psoriatic skin lesions, especially above the middle layer of the epidermis. Increased PRDX2 and GSTP1 may play a role in reduction of ROS-stress in cells, thereby preventing cells from ROS-induced DNA damage or cell death.

Taken together, this study showed an association of 36 identified proteins with biological regulation, including regulation of cell death (apoptosis), defense response, inflammatory response, and ROS regulation. Up-regulation of PRDX2 and GSTP1 may play roles in compensating mechanisms for reduction of ROS stress, and SFN may play roles in prevention of cancer development in proliferating cells through G2/M cell cycle arrest on accidental DNA damage within psoriatic skin lesions.

\section{Acknowledgements}

This study was supported by a grant from the KRIBB Research Initiative Program and 'The Happy tech. program through the National Research Foundation of Korea (NRF) funded by the Ministry of Education, Science and Technology (20100020765)'.

\section{References}

1. Shakery K and Reich K: Psoriasis - clinical picture and current therapy. Med Monatsschr Pharm 32: 335-344, 2009 (In German).

2. Nestle FO, Kaplan DH and Barker J: Psoriasis. N Engl J Med 361: 496-509, 2009.

3. Zippin JH: The genetics of psoriasis. J Drugs Dermatol 8: 414-417, 2009.

4. Zhang XJ, Huang W, Yang S, et al: Psoriasis genome-wide association study identifies susceptibility variants within LCE gene cluster at 1q21. Nat Genet 41: 205-210, 2009.

5. Reich A and Szepietowski J: Genetic and immunological aspects of the pathogenesis of psoriasis. Wiad Lek 60: 270-276, 2007 (In Polish).

6. Nickoloff BJ, Xin H, Nestle FO and Qin JZ: The cytokine and chemokine network in psoriasis. Clin Dermatol 25: 568-573, 2007.

7. Murphy M, Kerr P and Grant-Kels JM: The histopathologic spectrum of psoriasis. Clin Dermatol 25: 524-528, 2007.

8. Anderson KS, Wong J, Polyak K, Aronzon D and Enerback C: Detection of psoriasin/S100A7 in the sera of patients with psoriasis. Br J Dermatol 160: 325-332, 2009.

9. Wolk K, Witte E, Wallace E, et al: IL-22 regulates the expression of genes responsible for antimicrobial defense, cellular differentiation, and mobility in keratinocytes: a potential role in psoriasis. Eur J Immunol 36: 1309-1323, 2006.

10. Rozieres A, Hennino A and Nicolas JF: TNF alpha in the physiopathology of psoriasis. Ann Dermatol Venereol 133: 174-180, 2006.

11. Shilov VN and Sergienko VI: Oxidative stress in keratinocytes as an etiopathogenetic factor of psoriasis. Bull Exp Biol Med 129: 309-313, 2000.

12. Rashmi R, Rao KS and Basavaraj KH: A comprehensive review of biomarkers in psoriasis. Clin Exp Dermatol 34: 658-663, 2009.

13. Zhou Q, Mrowietz U and Rostami-Yazdi M: Oxidative stress in the pathogenesis of psoriasis. Free Radic Biol Med 47: 891-905, 2009. 
14. Ioannou M, Sourli F, Mylonis I, et al: Increased HIF-1 alpha immunostaining in psoriasis compared to psoriasiform dermatitides. J Cutan Pathol 36: 1255-1261, 2009.

15. Nakai K, Yoneda K, Maeda R, et al: Urinary biomarker of oxidative stress in patients with psoriasis vulgaris and atopic dermatitis. $J$ Eur Acad Dermatol Venereol 23: 1405-1408, 2009.

16. Wojas-Pelc A and Marcinkiewicz J: What is a role of haeme oxygenase-1 in psoriasis? Current concepts of pathogenesis. Int J Exp Pathol 88: 95-102, 2007.

17. Chua RA and Arbiser JL: The role of angiogenesis in the pathogenesis of psoriasis. Autoimmunity 42: 574-579, 2009.

18. Racz E and Prens EP: Molecular pathophysiology of psoriasis and molecular targets of antipsoriatic therapy. Expert Rev Mol Med 11: e38, 2009.

19. Simonetti O, Lucarini G, Goteri G, et al: VEGF is likely a key factor in the link between inflammation and angiogenesis in psoriasis: results of an immunohistochemical study. Int J Immunopathol Pharmacol 19: 751-760, 2006.

20. Krueger JG and Bowcock A: Psoriasis pathophysiology: current concepts of pathogenesis. Ann Rheum Dis 64 (Suppl 2): S30-S36, 2005.
21. Hermeking $\mathrm{H}$, Lengauer C, Polyak $\mathrm{K}$, et al: 14-3-3 sigma is a p53-regulated inhibitor of G2/M progression. Mol Cell 1: 3-11, 1997.

22. Chan TA, Hermeking H, Lengauer C, Kinzler KW and Vogelstein B: 14-3-3Sigma is required to prevent mitotic catastrophe after DNA damage. Nature 401: 616-620, 1999.

23. Samuel T, Weber HO, Rauch P, et al: The G2/M regulator 14-33 sigma prevents apoptosis through sequestration of Bax. J Biol Chem 276: 45201-45206, 2001.

24. Briganti S and Picardo M: Antioxidant activity, lipid peroxidation and skin diseases. What's new. J Eur Acad Dermatol Venereol 17: 663-669, 2003.

25. Vanizor Kural B, Orem A, Cimsit G, Yandi YE and Calapoglu M: Evaluation of the atherogenic tendency of lipids and lipoprotein content and their relationships with oxidant-antioxidant system in patients with psoriasis. Clin Chim Acta 328: 71-82, 2003.

26. Okayama Y: Oxidative stress in allergic and inflammatory skin diseases. Curr Drug Targets Inflamm Allergy 4: 517-519, 2005. 